Documentation et bibliothèques

DOCUMENTATION BIBLIOTHEQUES

\title{
Le système vidéotex canadien Télidon
} TELIDON: the Canadian Videotex System El sistema videotexto canadiense Telidon

\section{Edith Healy}

Volume 31, numéro 3, juillet-septembre 1985

URI : https://id.erudit.org/iderudit/1052778ar

DOI : https://doi.org/10.7202/1052778ar

Aller au sommaire du numéro

\section{Éditeur(s)}

Association pour l'avancement des sciences et des techniques de la documentation (ASTED)

ISSN

0315-2340 (imprimé)

2291-8949 (numérique)

Découvrir la revue

Citer cet article

Healy, E. (1985). Le système vidéotex canadien Télidon. Documentation et bibliothèques, 31(3), 107-111. https://doi.org/10.7202/1052778ar

\section{Résumé de l'article}

Télidon réunit les caractéristiques d'un système de télétexte et de vidéotex. Il se distingue des autres systèmes vidéotex par le mode de codage et de stockage de l'information graphique. Ses possibilités et ses domaines d'application sont donnés et les projets canadiens, dont il fait l'objet, brièvement décrits ainsi que ses perspectives d'avenir en fonction du marché de masse et des marchés spécialisés. L'avenir de Télidon dépend davantage d'un contenu que de l'originalité de sa technologie. Il devrait intéresser davantage les bibliothécaires en tant que fournisseurs d'information.
Tous droits réservés (C) Association pour l'avancement des sciences et des techniques de la documentation (ASTED), 1985
Ce document est protégé par la loi sur le droit d'auteur. L'utilisation des services d'Érudit (y compris la reproduction) est assujettie à sa politique d'utilisation que vous pouvez consulter en ligne.

https://apropos.erudit.org/fr/usagers/politique-dutilisation/ 


\title{
Le système vidéotex canadien Télidon
}

\author{
Edith Healy* \\ Université de Montréal
}

Télidon réunit les caractéristiques d'un système de télétexte et de vidéotex. I/ se distingue des autres systèmes vidéotex par le mode de codage et de stockage de l'information graphique. Ses possibilités et ses domaines d'application sont donnés et les projets canadiens, dont il fait l'objet, brièvement décrits ainsi que ses perspectives d'avenir en fonction du marché de masse et des marchés spécialisés. L'avenir de Télidon dépend davantage d'un contenu que de l'originalité de sa technologie. II devrait intéresser davantage les bibliothécaires en tant que fournisseurs d'information.

\section{TELIDON: the Canadian Videotex System}

TELIDON offers the characteristics of a teletex and videotex system. It differs from other videotex systems because of its form of coding and of storage for graphics. Its possibilities and fields of application are listed, its related Canadian projects are briefly outlined as well as its prospects as regards to mass and specialized markets. The future of TELIDON rests more in its contents than in the originality of its technology. It should be of more interest to librarians as purveyors of information.

\section{El sistema videotexto canadiense Telidon}

Telidon reúne las características de un sistema de teletexto y de videotexto. Se diferencia de los demás sistemas videotextos por su modo de codificación y de almacenamiento de la información gráfica. El autor hace alusión a los proyectos canadienses de Telidon, a sus posibilidades y a sus campos de aplicación, así como a sus perspectivas futuras en función del mercado generaly de los mercados especializados. El porvenir de Telidon depende más del contenido que de la originalidad de su tecnología. Debería interesar en especial a los bibliotecarios como proveedores de información.
Télidon est un système télétexte-vidéotex de deuxième génération, développé par le Centre de Recherche du ministère des Communications. Télidon a été présenté au public en août 1978. Quatre ans plus tard, soit au moment de remettre la technologie à l'industrie canadienne, le gouvernement y avait investi plus de 41 millions de dollars. II a cependant continué de participer à plusieurs projets au-delà de cette période.

Bien que les deux termes "vidéotex" et "télétexte» soient parfois considérés comme des synonymes, la majorité des auteurs y apportent la distinction suivante:

- le vidéotex est un service de recherche d'information interactif, à accès public, qui utilise comme récepteur un téléviseur modifié, relié à un ordinateur par un lien tangible qui peut être une ligne téléphonique, le câble, des fibres optiques, un satellite... II s'agit d'un système bidirectionnel, c'est-à-dire que le message de l'utilisateur est transmis à l'ordinateur qui l'analyse et y répond;
- le télétexte est un système unidirectionnel qui utilise comme mode de transmission le câble de télévision. Un nombre restreint de pages d'informations est diffusé à intervalles réguliers selon un ordre prédéterminé. Les pages peuvent être sélectionnées et affichées sur l'écran. Le décodeur capte les pages choisies, au moment où elles passent, les garde en mémoire et les affiche sur l'écran. Le client doit attendre la diffusion de la page voulue, ce qui implique des limites quant au nombre de pages inscrites dans la banque de données. Par exemple, si la diffusion se fait sur un canal entier, un cycle de 12 secondes est nécessaire pour diffuser 10000 pages. Par contre, si la diffusion se fait seulement à partir des lignes inutilisées d'un signal de télévision, il faut 12 secondes pour 100 pages. Or, on sait que les usagers ont tendance à perdre patience si l'attente se prolonge au delà de 5 à 6 secondes. Les banques de données sont donc généralement limitées à 100 pages d'information'. Télidon peut être utilisé selon l'un ou l'autre mode. 
La conception des systèmes vidéotex débute vers les années 1970 et correspond à la naissance du concept de la société d'information ou société câblée. C'est aussi durant cette période que se développent les banques de données accessibles sur des ordinateurs qui sont de plus en plus puissants et de moins en moins chers. Cependant, les banques de données étaient limitées, quant à leur accès, à des spécialistes qui devaient avoir une certaine connaissance des procédures d'interrogation. Le vidéotex a été conçu pour répondre aux besoins de l'homme "ordinaire» afin de lui offrir un accès facile à l'information, via son propre terminal, à la maison.

\section{Caractéristiques de Télidon}

Au moment de l'annonce de Télidon, d'autres systèmes de vidéotex existaient, notamment le système de la Grande-Bretagne, Prestel et celui de la France, Antiope.

La différence fondamentale entre Télidon et ces systèmes se situe au niveau du mode de codage et de stockage de l'information graphique ${ }^{2}$.

Télidon utilise le mode alphagéométrique, contrairement aux systèmes de première génération qui emploient le mode alphamosaïque. On dit en fait que Télidon est un code abrégé pour le stockage et la transmission rapide de graphiques. Sept codes, appelés Instructions de Description d'Images (IDI) sont utilisés pour décrire une image. II suffit de transmettre ces instructions pour la dessiner.

Les modes d'affichages conventionnels (alphamosaïques) sont limités à une définition de 60 lignes par 80 caractères (4800 éléments) alors que Télidon permet une résolution de 76800 éléments sur un écran de télévision et peut aller jusqu'à 1228800 éléments sur un écran de haute résolution ${ }^{3}$. La faible résolution du procédé en mosaïque provient du fait que chaque élément de la matrice doit être défini, ce qui est très long. Avec Télidon, une image complexe peut être décrite avec 3000 bytes seulement. Outre le fait que le mode de codage de Télidon offre une meilleure résolution de l'image, son avantage le plus important est qu'il permet une variation de résolution, dépendamment de la qualité de l'écran ou du terminal utilisé. Cependant, peu importe le degré de perfectionnement des terminaux, tous peuvent recevoir et interpréter les $\mathrm{IDI}^{4}$. Seule la qualité de l'image est affectée. Au contraire, les images composées selon le mode mosaïque sont toujours reproduites de la même façon, peu importe la qualité du récepteur.

Autrement dit, Télidon s'appuie sur un protocole de communication indépendant de la représentation, du mode de transmission ainsi que du contenu de l'image. Télidon est compatible autant avec les technologies futures qu'avec les technologies antérieures, ce qui en fait un système très souple ${ }^{5}$.

Du point de vue technique, Télidon est donc de loin en avance sur ses concurrents. De nombreux experts en communication le considèrent comme le meilleur système de vidéotex au monde ${ }^{6}$. II n'est donc pas surprenant que son mode de codage ait été accepté comme norme vidéotex alphagéométrique mondiale?

Télidon offre aussi une vaste gamme de couleurs (16,8 millions de teintes, selon la norme NAPLPS) et permet de faire de l'animation et de la superposition multiple.

\section{Potentiel du système et ses applications}

Comme l'ont souligné plusieurs auteurs ${ }^{8}, 9$, la technologie du vidéotex semble être à la recherche d'un marché. II est, encore actuellement, plus facile de parler de ce que peut faire le vidéotex que de ce qu'il fait. Autrement dit, on connaît bien les possibilités du contenant mais on ignore encore quel contenu y mettre.

Télidon offre d'immenses possibilités, dont les seules limites sont, semble-t-il, liées à notre manque d'imagination. En ce sens, Télidon fait face au même problème que toute nouvelle technologie. C'est ce que McLuhan ${ }^{10}$ appelle «the rear view effect", c'est-à-dire la réaction à considérer une nouvelle technologie en fonction des rôles et des attributs que nous lui connaissons. Ainsi, le vidéotex a été perçu comme une nouvelle façon d'afficher les informations traditionnellement trans-
2. Andrej Tenne-Sens, «Telidon graphical and library associations», Information Technology and Libraries, vol. 1, no. 2 (June 1982), 98-110.

3. H.G. Bown, C.D. O'Brien, W. Sawchuck and J.R. Storey, «A general description of Telidon: a Canadian proposal for videotex systems", Communication Research Center/Ministère des Communications CRC Technical Note, no. 697 E, Ottawa (December 1978).

4. Wolfe, Videotex: the new television-telephone information services. London, Heyden, c1980, XII, $170 \mathrm{p}$

5. Lare Mischo and Kevin Hegarty, "Videotex - The library of the future", Information Technology and Libraries, vol. 1, no. 3 (September 1982), 276-277.
6. Andrej Tenne-Sennes, notamment.

7. R.V. Baser, Telidon, l'expérience vidéotex canadienne. Présenté à la $5^{\text {ième }}$ table ronde de la FIA, 28 avril 1982. Casablanca, Maroc.

8. Ken Jensen and A.J.S. Ball, «A novelty whose time has come», Canadian Library Journal, vol. 38, no. 4 (August 1981), 199-205.

9. David Wright, "Une technologie de pointe à la recherche d'un marché», In Search / Enquête, vol. 6, no 1 (hiver 1979).

10. David Godfrey, The Telidon book. Toronto, Press Porcepic, $1981,300 \mathrm{p}$. 
mises sous forme papier. II est de plus en plus évident que cette conception du vidéotex ne s'ajuste pas à la réalité. Télidon est avant tout un moyen de communication visuel, qui devrait trouver sa place dans de multiples applications où les informations changent rapidement.

En fonction du marché initial visé, le marché de masse, plusieurs questions peuvent être posées:

Quelle est l'information désirée par les usagers ? Est-ce que les usagers sont diposés à lire sur un écran comme sur un support imprimé ?

Le vidéotex peut-il créer sa propre information ?

De quelle façon l'information doit-elle être organisée?

Alors que la majorité des gens semblent tradionnellement réticents à payer pour de l'information, quel prix les usagers sont-ils prêts à payer pour l'information ?"11

La réponse à ces questions pourrait permettre de mieux cerner les applications possibles et souhaitées du système Télidon. L'éventail des applications est large : recherche d'informations volatiles et "compactes", qui tirent leur valeur dans leur immédiateté: services de télémagasinage, services de transactions bancaires, réservation de billets d'avion, de chambres d'hôtels, courrier électronique, jeux électroniques, cours de formation, enseignement assisté par ordinateur, diffusion de logiciels, etc...

De nombreux projets-pilotes, la plupart sous la dépendance des compagnies de téléphone, ont été mis sur pied pour prendre le pouls de la population quant à ce nouveau média de communication.

Plusieurs de ces projets ont pris fin à l'heure actuelle. Comme les terminaux et les informations sont offerts gratuitement au cours de ces essais, il est difficile de prévoir la viabilité des systèmes expérimentés. Très peu sont offerts sur une base commerciale.

\section{Systèmes commerciaux}

\section{1) Grassroots}

La banque de données Grassroots a été produite par un des plus grands éditeurs électroniques: Informat. ll s'agit d'une banque spécialisée sur les services agricoles. Elle s'adresse à environ 2000 abonnés du sud du Manitoba, de la Saskatchewan et de l'Ontario' ${ }^{12}$. Les abonnés ont aussi accès à d'autres services (opérations bancaires, télémagasinage, aide aux consom- mateurs, services éducatifs, contes pour enfants...). C'est le premier service commercial Télidon instauré au Canada. II offre quotidiennement plus de 48000 pages d'information fournies par environ 270 fournisseurs d'information qui l'alimentent à raison de $2 \$$ par page par mois.

En 1984 , il en coûtait $59 \$$ par mois pour louer l'équipement et $05 \mathrm{C}$ par minute pour le temps de connexion. Le fait qu'il n'y ait eu aucun abonnement annulé depuis au moins six mois atteste que le service est apprécié ${ }^{13}$.

\section{2) Téléguide-Ontario}

II s'agit d'une banque de données conçue pour les touristes et offerte gratuitement aux usagers. Environ 400 terminaux publics ont été installés (on en prévoyait 1500 ) dans les gares, les halls d'hôtels... La banque contient des informations touristiques sur le logement, les restaurants, les magasins, les divertissements, des données météorologiques, etc.

Ce projet a nécessité un investissement de 14 millions de dollars (cinq millions du gouvernement ontarien, sept d'Infomart et deux du gouvernement fédéral).

Les objectifs de ce projet étaient très ambitieux: réduire le prix de l'équipement et les logiciels en augmentant le volume du marché; maximiser l'exposition du public à Télidon et créer un marché de masse.

\section{3) Novatex}

Le système Novatex de Téléglobe Canada est un service international d'informations d'affaires. La banque de données comprend plus de 10000 pages de nouvelles générales et financières, d'informations touristiques et gouvernementales, d'informations agricoles et sur les ressources naturelles, des informations statistiques et boursières.

Lancé en janvier 1981, le service a été offert sur une base commerciale à partir de janvier 1984 jusqu'à juillet 1984. II s'adressait aux entreprises et aux gestionnaires de sociétés multinationales. II en coûtait $10 \$$ par heure d'utilisation et $50 \$$ par mois pour le service de base. Un progiciel d'informations boursières était disponible à raison de $100 \$$ par mois.
11. Mary Nash, « Videotex versus online bibliographic database: a Canadian Perspective», Online Review, vol. 6, no. 4 (August 1982), 291-296.

12. Maryanne Petrovich, Grassroots: farmers embrace information age , Videotex World, vol. 1 , no. 1 (September 1984), 5-9.
13. Peter Dolan, "Grassroots: Videotex bellwether», Videotex Canada, (Spring 1983), 18-20. 


\section{Systèmes publics}

\section{1) Cantel}

Cantel est une banque de données Télidon du gouvernement du Canada élaborée par un groupe de travail du ministère des Approvisionnements et Services. Infomart en assure la gestion. Son objectif est d'améliorer l'accès du public aux programmes et services fédéraux. Elle contient environ 55000 pages d'information (en anglais et en français). C'est la plus grande banque gouvernementale offerte au public. On peut y trouver toutes sortes d'informations comme, par exemple, comment se procurer un passeport, les droits des consommateurs, les attractions touristiques, des renseignements pratiques sur l'emploi et des documents techniques dans des domaines spécialisés.

Trente kiosques Cantel sont installés dans 14 villes (centres commerciaux, bibliothèques, bureaux de poste...).

\section{2) Vista}

Vista est le fruit de la collaboration de Bell Canada et du ministère des Communications. Ce projet constitue le plus grand essai Télidon: plus de 500 terminaux ont été installés dans des foyers et des endroits publics.

Vista offrait l'accès à environ 65000 pages d'informations (services bancaires, informations touristiques, télémagasinage....). L'essai a pris fin en septembre 1983. Parmi les fournisseurs, mentionnons: Le Soleil, Édimedia, Télédirect (pages jaunes), Statistique Canada et I'Université du Québec.

\section{3) IRIS}

Ce projet de la Société Radio-Canada (qui a débuté en avril 1983 et s'est poursuivi jusqu'à décembre de la même année) visait l'introduction des services nationaux de vidéotex diffusés en mode télétexte.

Environ 200 foyers ont été équipés de terminaux leur donnant accès au magazine IRIS (nouvelles nationales, régionales et locales, loisirs ...) constitué d'environ 250 pages régulièrement mises à jour.

\section{Perspectives d'avenir de Télidon}

Les perspectives d'avenir de Télidon doivent, à notre avis, être considérées en fonction de deux marchés très différents, le marché de masse et le marché des petits groupes spécialisés.

\section{Marché de masse}

De toute évidence, Télidon n'a pas envahi le marché comme on s'y attendait. En 1983, on prévoyait qu'il y aurait 30000 terminaux NAPLPS en Amérique du Nord à la fin de 1984. Or, on estime, en 1984 , ce nombre à $10000^{14}$. On peut cependant se consoler en se disant qu'il n'y a pas que Télidon qui éprouve des difficultés pour percer le marché. En effet, le vidéotex américain, Viewtron, $n^{\prime}$ a pas, lui non plus, atteint ses objectifs ${ }^{15}$.

On continue cependant à être optimiste. Le ministère des Communications prévoit que Télidon sera accessible dans les foyers d'ici un à deux ans. Les experts-conseils chiffrent entre un à quatre millions le nombre de terminaux vidéotex qui pourraient être installés au Canada d'ici la fin de la décennie ${ }^{16}$. Il s'agit ici de prévisions assez conservatrices puisque le ministère des Communications parle de 10 millions, dans son diaporama sur Télidon... Aux Ėtats-Unis, on croit que près de $30 \%$ des foyers pourraient posséder un système vidéotex d'ici 199017 .

Bien qu'optimiste, on s'interroge sur les causes pouvant expliquer le fait que Télidon n'aie pas encore envahi le marché. Les facteurs les plus souvent invoqués sont les suivants:

1 - le prix de l'équipement est encore trop élevé (les décodeurs se vendaient 2300 \$ en 1981; actuellement, on peut se les procurer à partir de 800 \$);

2- le manque de contenu;

3- I'organisation de l'information dans les banques et les façons d'y avoir accès sont encore jugées peu satisfaisantes. (On travaille actuellement sur d'autres modes d'accès comme la sélection du menu par simple toucher de l'écran ou par reconnaissance de la voix...)

En ce qui concerne les deux premiers facteurs, on peut dire que Télidon fait face à l'éternel problème de l'oeuf et de la poule: les prix sont très dépendants du volume des ventes alors que le marché potentiel est limité par le prix.

De la même façon, en ce qui a trait au contenu, on peut dire que nul ne désire investir dans le matériel avant que les services ne soient disponibles tandis que d'autre part les fournisseurs
14. Martin Lane, "Confessions of a prognosticator", Videotex World, vol. 1, no. 2 (Décember 1984), 6-9, 28.

15. "Viewtron eyes PC Market, Denis Doomsday rumors», Information Today, vol. 2, no. 1 (January 1985), 1-8.
16. R.V. Baser, Télidon, l'expérience...

17. Lilly Trabusco, «Teleshopping: will your TV set become the new department store?» Bulletin of the American Society for Information Science, vol. 8, no. 3 (February 1982), 14-17. 
hésitent à créer des services lorsque les auditoires dotés du matériel nécessaire sont restreints ${ }^{18}$.

Un nouvel élément pourrait cependant avoir un impact positif sur l'entrée du vidéotex dans les foyers: la venue des micro-ordinateurs domestiques. En effet, il existe maintenant des logiciels de décodage qui permettent d'utiliser le microordinateur comme terminal vidéotex. De tels logiciels sont disponibles pour l'Apple, le Commodore 64, I'IBM, le DEC Rain-Bow, le Sony SMC-70... Ces logiciels sont peu coûteux (certains à $100 \$$ ou 200 \$). Cependant, il semble que le marché des micro-ordinateurs domestiques ne soit pas très florissant. IBM annonçait récemment qu'elle cessait la production du IBM junior... En outre, le micro-ordinateur ne donne pas encore accès aux banques de données grand-public diffusées pas câble.

II reste quand même un espoir: Norpak serait sur le point de signer avec un des principaux manufacturiers d'appareils de télévision, une entente prévoyant la mise en marché d'appareils munis d'un décodeur intégré pour un coût majoré d'environ $100 \$ 19$.

En ce qui concerne les services de vidéotex destinés à un usage domestique, il est probable que la transmission se fera par câble. Vidéotron pourrait donc en devenir le serveur. II sera intéressant de surveiller de près le projet pilote de Vidéotron, projet qui doit normalement débuter en septembre 1985, au cours duquel 250 terminaux seront installés dans les foyers de la région de Montréal. Les résultats de ce projet pourraient bien déterminer l'avenir de Télidon en tant que système d'information destiné au grand public...

\section{Marchés spécifiques: \\ Closed Groupes Users (CGU)}

II semble de plus en plus évident que c'est d'abord vers ces marchés que s'orientera la technologie du vidéotex Télidon, comme l'a fait Prestel (près de $85 \%$ des usagers de Prestel proviennent du milieu des affaires ${ }^{20}$.

Les petits groupes bien spécifiques, dont les individus partagent les mêmes intérêts et ont les mêmes besoins, comme le milieu des affaires, représentent une clientèle idéale puisque les banques de données peuvent être plus restreintes, permettant alors aux fournisseurs d'informations de couvrir leurs frais même si l'audience est limitée. Au contraire, si on s'adresse à un public diversifié, la banque de données doit être générale et volumineuse. L'audience doit, par conséquent, être grande pour intéresser les fournisseurs d'informations.

Selon un représentant de la compagnie Formic (qui distribue l'équipement pour les micro-ordinateurs et qui agit aussi comme serveur) Télidon connaît une popularité croissante auprès des $P M E$ et en particulier auprès des milieux d'affaires. Évidemment, pour ces marchés, le facteur "prix» est beaucoup moins déterminant qu'il ne l'est pour le public en général...

\section{Conclusion}

L'avenir de Télidon est très incertain. Comme le disait le ministre des Communications Francis Fox: «le défi pour Télidon à ce moment n'est plus au niveau de la technologie elle-même, mais à celui du contenu»21. II faut donc trouver des moyens pour encourager les fournisseurs d'informations.

Selon Lane 22 , les manufacturiers canadiens ont déjà concédé le marché de l'équipement aux États-Unis et si la tendance se poursuit, il en sera de même pour le marché des logiciels et des contenus.

Ainsi que le fait Pierre Juneau ${ }^{23}$, on peut se poser la question suivante: Pouvons-nous établir une industrie d'information économiquement viable au Canada ou la technologie que nous avons développée sera-t-elle exploitée par des entreprises étrangères? Autrement dit: Have we sold a better mouse trap to the world only to find that we have become the mice ? ${ }^{24}$

En tant que professionnel de l'information, nous pouvons nous demander quelle est la place du bibliothécaire dans le développement de cette technologie. Allons-nous encore une fois laisser à d'autres le soin de développer un nouvel outil de téléréférence? N'avons-nous pas un rôle important à jouer en tant que fournisseur d'information? Faut-il se surprendre que seulement deux ou trois des 66 membres de l'Association des Fournisseurs d'Informations pour le vidéotex soient associés à des bibliothèques? Et si, au-delà de toutes prévisions, Télidon entrait dans les foyers, ne devrionsnous pas dès maintenant nous engager pour intervenir dans le contenu de l'information et pour favoriser ainsi son accès?
18. Douglas, F. Parkhill, «Les progrès sur la scène canadienne». Document présenté à la conférence et à l'exposition internationale Viewdata 81 , du 6 au 8 octobre 1981, à Londres.

19. Affaires extérieures du Canada, "Videotex/Teletex: the new users", Videotex World, vol. 1 no. 2 (December 1984), s1-s8.

20. Mary M. Nash et M. Janice Pereira, «Canadian scene: Telidon and library application», Canadian Library Journal, vol. 39, no. 4 (August 1982), 249-254
21. «ntrevue exclusive avec le ministre des Communications du Canada, I'honorable Francis Fox", Informatique et bureautique, vol. 3 no 9 (Novembre 1982), 10-16.

22. Martin Lane, «Three years on the merry-go-round", Videotex Canada, vol. 2, no. 1 (Summer 1983), 33-37.

23. Pierre Juneau, «Videotex across Canada: Where do we go from here?», Videotex Canada, vol. 1 (Spring 1982), 5-6, 17-19.

24. Scott Gardiner, cité par Pierre Juneau. 